\title{
CONVEXITY AND CYLINDRICAL ESTIMATES FOR MEAN CURVATURE FLOW IN THE SPHERE
}

\author{
HUY THE NGUYEN
}

Abstract. We study mean curvature flow in the sphere with the quadratic curvature condition $|A|^{2} \leq \frac{1}{n-2} H^{2}+4 K$ which is related to but different from two-convexity for submanifolds of the sphere. We classify type $I$ singularities with no further hypotheses. If $H>0$, then we apply the Huisken-Sinestrari convexity estimates to this situation and show that we can classify type $I I$ singularities. This shows that at a singularity the surface is asymptotically convex. We then prove cylindrical estimates for the mean curvature flow and a pointwise gradient estimate which shows that near a singularity the surface is quantitatively convex.

\section{INTRODUCTION}

Let $X_{0}: \mathcal{M}^{n} \rightarrow \mathbb{S}^{n+1}$ be a smooth immersion of an $n$-dimensional hypersurface into the round sphere, $n \geq 4$. Then the mean curvature flow is the gradient flow of the area functional given by the partial differential equation

$$
\begin{aligned}
\frac{\partial}{\partial t} X(p, t) & =-H(p, t) \nu(p, t), \quad p \in \mathcal{M}, t \geq 0, \\
X(p, 0) & =X_{0}(p)
\end{aligned}
$$

where $-H(p, t) \nu(p, t)=\vec{H}(p, t)$ is the mean curvature. For a compact hypersurface of the sphere, there is no preferred notion of inside and outside, the mean curvature flow is chosen simply to decrease the area functional.

Geometric flows have recently been very successful in proving sphere theorems, that is, restrictions on the curvature which give a classification of the manifolds. In fact recent results suggest that such classifications can be a starting point in proving generalised sphere theorems or connected sum theorems, for example

Theorem 1.1 (8]). Any smooth closed n-dimensional two-convex immersed surface $X_{0}: \mathcal{M}^{n} \rightarrow \mathbb{R}^{n+1}$ with $n \geq 3$ is diffeomorphic either to $\mathbb{S}^{n}$ or to a finite connected sum of $\mathbb{S}^{n-1} \times \mathbb{S}^{1}$.

The idea is that by relaxing the convexity condition to a flexible condition, in particular a curvature condition that is closed under connected sum, we obtain a connected sum theorem. Another example is positive scalar curvature for threemanifolds

Theorem $1.2([1])$. Let $\mathcal{M}^{3}$ be a compact Riemannian manifold with positive scalar curvature. Then $\mathcal{M}^{3}$ is diffeomorphic to $\mathbb{S}^{3}$ or a finite connected sum of $\mathbb{S}^{2} \times \mathbb{S}^{1}$ and $\mathbb{S}^{2} \tilde{\times} \mathbb{S}^{1}$.

Received by the editors July 8, 2011 and, in revised form, July 19, 2012.

2010 Mathematics Subject Classification. Primary 53C44.

The author was supported by The Leverhulme Trust. 
The proof of both these theorems use geometric flows, the mean curvature flow and the Ricci flow respectively. They are respectively generalisations of the following theorems.

Theorem $1.3([3])$. Any smooth closed $n$-dimensional convex immersed surface $X_{0}: \mathcal{M}^{n} \rightarrow \mathbb{R}^{n+1}$ with $n \geq 2$ which flows by mean curvature, converges in finite time to a round point.

and

Theorem $1.4([2])$. Let $\mathcal{M}^{3}$ be a compact three-manifold with positive Ricci curvature $R_{i j} \geq K g_{i j}$; then under Ricci flow, positive Ricci curvature is preserved and converges in finite time to a spherical space form.

The idea of both proofs is to analyse the formation of singularities, and prove convexity estimates or Hamilton-Ivey estimates. Such estimates show that as we approach a singularity, we enter the boundary cases to the sphere theorems above.

In this paper, we will study mean curvature flow in the sphere, $\mathbb{S}_{K}^{n}$, under the following assumption:

$$
|A|^{2}<\frac{1}{n-2} H^{2}+4 K, H>0 .
$$

If the first inequality holds, we say that the hypersurface is quadratically bounded. If the second condition holds, it is called mean convexity. If both inequalities hold we say that the surface is mean convex quadratically bounded. This is related to a rigid curvature cone, introduced by Huisken,

Theorem $1.5([5])$. Let $n \geq 3$ and $\mathcal{N}^{n+1}$ be a spherical space form of sectional curvature $K$. Let $\mathcal{M}_{0}$ be a compact hypersurface without boundary which is smoothly immersed in $\mathcal{N}$ and suppose that we have on $\mathcal{M}_{0}$

$$
|A|^{2}<\frac{1}{n-1} H^{2}+2 K
$$

Then either the mean curvature flow exists for finite time $t \in[0, T)$ and $\mathcal{M}_{t}$ converges to a round point as $t \rightarrow T$ or exists for infinite time and converges to a great sphere.

With the assumption of mean convexity it is well known that the flow will exist only for finite time and hence the first alternative must hold, that is, we converge to a round point.

In this paper, we will be concerned with the analysis of singularities of the mean curvature flow of hypersurfaces which are mean convex quadratically bounded.

The paper is divided as follows. In section two, we recall the evolution equations and hypersurface equations that will be required in the following.

In section three, we show that the quadratic curvature condition is preserved by the mean curvature flow.

Then in section four we study type $I$ singularities and show that under the quadratic condition we can classify the blow up limits, without the hypothesis of mean convexity.

In section five, we adapt the Huisken-Sinestrari convexity estimates from Euclidean space to the round sphere. 
Theorem 1.6. For a smooth closed mean convex hypersurface $\mathcal{M}^{n} \subset \mathbb{S}^{n+1}$, for all $\eta>0$ there exists $C_{\eta}\left(n, \mathcal{M}_{0}\right)$ such that for the solution of the mean curvature flow $\mathcal{M}_{t}$ we have the following bound for the eigenvalue $\lambda_{1}$ of the second fundamental form:

$$
\lambda_{1} \geq-\eta H-C_{\eta} .
$$

As the $\eta>0$ is arbitrary, this implies that any surface obtained by a type $I I$ rescaling about a singularity is weakly convex. As the proof is very similar to the Euclidean case, we will only point out the minor differences in the case of $\sigma_{2}$.

In section six, we prove a cylindrical estimate for mean curvature flow for mean convex and quadratically bounded hypersurfaces. This shows that at points where $\lambda_{1}$ is small, the curvature is close to the curvature of a cylinder.

Theorem 1.7. For a given smooth, closed hypersurface $\mathcal{M}_{0} \subset \mathbb{S}^{n+1}$ that satisfies (1.1), then for any $\eta>0$ there exists $C_{\eta}$ such that

$$
\left|\lambda_{1}\right| \leq \eta H \Longrightarrow\left|\lambda_{i}-\lambda_{j}\right|^{2} \leq c(n) \eta H^{2}+C_{\eta}, \quad \forall i, j \geq 2 .
$$

In section seven, we prove a pointwise gradient estimate. This allows us to compare curvature at different points of surface.

Theorem 1.8. For a given smooth, closed hypersurface $\mathcal{M}_{0} \subset \mathbb{S}^{n+1}$ that satisfies (1.1), then for a solution of the mean curvature flow $\mathcal{M}_{t}$ there exists $c=$ $c\left(n, \mathcal{M}_{0}\right), C=C\left(n, \mathcal{M}_{0}\right)$ such that

$$
|\nabla A|^{2} \leq c|A|^{4}+C
$$

everywhere on $\mathcal{M} \times[0, T)$.

Note that this gradient estimate differs from the standard ones as it only depends on the curvature at a point. The standard estimates depend on the maximum of the curvature over the entire manifold.

The author would like to thank the anonymous referee whose careful reading and useful comments improved the presentation and exposition of this paper.

\section{Preliminaries}

In this section we will gather together various facts about quadratically bounded surfaces and notation that we will require later on.

2.1. Submanifolds. Here we state several well-known equations for submanifolds of $\mathbb{S}^{n}$.

Proposition 2.1 (Curvature formulae). Let $\mathcal{M}^{n} \subset \mathbb{S}^{n+1}$ be a hypersurface of the round sphere. Then we have the following equations:

$$
\begin{aligned}
R_{i j k l} & =\left(\bar{g}_{i k} \bar{g}_{j l}-\bar{g}_{i l} \bar{g}_{j k}\right)+h_{i k} h_{j l}-h_{i l} h_{j k}, \\
R_{i k} & =(n-1) \bar{g}_{i k}+\left(H h_{i k}-h_{i p} h_{j}^{p}\right), \\
R & =n(n-1)+H^{2}-\sum_{i j} h_{i j}^{2}=n(n-1)+H^{2}+A^{2},
\end{aligned}
$$

Simons identity,

$$
\triangle h_{i j}=\nabla_{i} \nabla_{j} H+\left(n-A^{2}\right) h_{i j}+H\left(h_{i p} h_{j}^{p}-\bar{g}_{i j}\right) .
$$

We also have the following consequence of the Codazzi inequality. 
Lemma 2.2. For any hypersurface $\mathcal{M}_{0} \subset \mathbb{S}^{n+1}$ we have that

$$
|\nabla A|^{2} \geq \frac{3}{n+2}|\nabla H|^{2} .
$$

2.2. Evolution equations. In this section we gather together all the evolution equations that we will be using in this paper.

Proposition $2.3([5])$. Let $X_{0}: \mathcal{M}^{n} \rightarrow \mathbb{S}^{n+1}$ be an embedding of a compact hypersurface in $\mathbb{S}^{n+1}$. Then for the mean curvature flow, we have the following evolution equations:

$$
\begin{aligned}
\partial_{t} X & =-H \nu \\
\partial_{t} g_{i j} & =-2 H h_{i j}, \\
\partial_{t} h_{i j} & =\triangle h_{i j}-2 H h_{i}^{p} h_{p j}+|A|^{2} h_{i j}+2 K H g_{i j}-n K h_{i j}, \\
\partial_{t} H & =\triangle H+H\left(|A|^{2}+n K\right), \\
\partial_{t}|A|^{2} & =\triangle|A|^{2}-2|\nabla A|^{2}+2|A|^{2}\left(|A|^{2}+n K\right)-4 n K\left(|A|^{2}-\frac{H^{2}}{n}\right), \\
\partial_{t} H^{2} & =\triangle H^{2}-2|\nabla H|^{2}+2 H^{2}\left(|A|^{2}+n K\right), \\
\partial_{t} \Gamma_{j k}^{i} & =-g^{i l}\left(\nabla_{j}\left(H h_{k l}\right)+\nabla_{k}\left(H h_{j l}\right)-\nabla_{l}\left(H h_{j k}\right)\right)=A * \nabla A .
\end{aligned}
$$

In particular, using the evolution equation of the Christoffel symbol, together with the evolution equation of the evolution of the second fundamental form, we can derive the evolution equation of the norm of the derivative of the second fundamental form as in [2, $§ 13]$,

$$
\frac{\partial}{\partial t}|\nabla A|^{2}-\triangle|\nabla A|^{2} \leq-2\left|\nabla^{2} A\right|^{2}+c_{n}|A|^{2}|\nabla A|^{2}+d_{n}|A||\nabla A|^{2} .
$$

\section{Curvature cones}

In this section, we will show that the curvature cone (1.1) is preserved by the mean curvature flow.

3.1. Quadratic curvature cones. If we assume that the quadratic curvature inequality is strict, that is,

$$
|A|^{2}<\frac{1}{n-2} H^{2}+4 K
$$

then there exists an $\varepsilon$ such that the following inequality holds everywhere on $\mathcal{M}_{0}^{n} \subset$ $\mathbb{S}_{K}^{n+1}[5]$ :

$$
|A|^{2} \leq \frac{1}{n-2+\varepsilon} H^{2}+2(2-\varepsilon) K .
$$

Let $\beta_{n}=2(2-\varepsilon)$ and $\alpha_{n}=\frac{1}{n-2+\varepsilon}$; then we have the following theorem.

Theorem 3.1 ([4]). Consider the curvature inequality

$$
|A|^{2} \leq \alpha_{n} H^{2}+\beta_{n} K
$$

which holds everywhere on $\mathcal{M}_{0}$. This inequality is preserved on $\mathcal{M}_{t}$ by the mean curvature flow for all times $0 \leq t \leq T \leq \infty$ where the solution exists. 
Proof. Consider the following evolution equation:

$$
\begin{aligned}
\partial_{t}\left(|A|^{2}-\alpha_{n} H^{2}\right) & =\triangle\left(|A|^{2}-\alpha_{n} H^{2}\right)-2\left(|\nabla A|^{2}-\alpha_{n}|\nabla H|^{2}\right)+2 \beta_{n} K\left(|A|^{2}+n K\right) \\
& +2\left(|A|^{2}-\alpha_{n} H^{2}-\beta_{n} K\right)\left(|A|^{2}+n K\right)-4 n K\left(|A|^{2}-\frac{1}{n} H^{2}\right),
\end{aligned}
$$

where we used the fact that $\alpha_{n}=\frac{2}{2 n-\beta_{n}}$. In addition, we have the inequality,

$$
\begin{aligned}
& 2 \beta_{n} K\left(|A|^{2}+n K\right)-4 n K\left(|A|^{2}-\frac{1}{n} H^{2}\right) \\
= & 2 K\left(\left(\beta_{n}-2 n\right)|A|^{2}+2 H^{2}+\beta_{n} n K\right) \\
= & -2 K\left(2 n-\beta_{n}\right)\left(|A|^{2}-\frac{2}{2 n-\beta_{n}} H^{2}-\frac{n \beta_{n}}{2 n-\beta_{n}} K\right) .
\end{aligned}
$$

Now as $\beta_{n}=2(2-\epsilon)$ we have that $\frac{n}{2 n-\beta_{n}} \leq 1$ and $\frac{2}{2 n-\beta_{n}} \leq \frac{3}{n+2}, n \geq 4$. By applying (2.1) and the parabolic maximum principle, this proves the theorem.

3.2. Rigidity. The quadratic bound is optimal for connected sum theorems in dimensions $n \geq 4$. Consider the tori $M^{k, n-k}(r, s)=\mathbb{S}_{r}^{k} \times \mathbb{S}_{s}^{n-k}$ with $r^{2}+s^{2}=1$. This has second fundamental form, with eigenvalues $\lambda$ with multiplicity $k$ and $\mu$ with multiplicity $n-k$ and $\lambda \mu=-1$. Without loss of generality, for the case $k=1$ we may assume that $\lambda=-\frac{s}{r}, \mu=\frac{r}{s}$. We have the following formulae:

$$
\begin{aligned}
|A|^{2} & =\frac{(n-1) r^{4}+s^{4}}{r^{2} s^{2}}, \\
H & =\frac{(n-1) r^{2}-s^{2}}{r s}, \\
H^{2} & =\frac{\left((n-1) r^{2}-s^{2}\right)^{2}}{r^{2} s^{2}} .
\end{aligned}
$$

The minimal tori then occurs when $n-1=\frac{s^{2}}{r^{2}}$ or $s^{2}=\frac{n-1}{n}$. This implies the following equation for such estimates:

$$
|A|^{2}=n .
$$

As a minimal submanifold, it is stationary under the mean curvature flow and hence cannot improve. However it lies outside our curvature cone considered in 3 . because it satisfies

$$
|A|^{2}-\frac{1}{n-1} H^{2}-2 K=\frac{(n-1)}{(n-2)} \frac{s^{2}}{r^{2}} .
$$

If $k=2$ we have that

$$
\begin{aligned}
H & =-2 \frac{s}{r}+(n-2) \frac{r}{s} \\
& =\frac{(n-2) r^{2}-s^{2}}{r s} .
\end{aligned}
$$

So we have

$$
\begin{aligned}
H^{2} & =\frac{(n-2) r^{4}+4 s^{4}-4(n-2) r^{2} s^{2}}{r^{2} s^{2}} \\
|A|^{2} & =\frac{2 s^{4}+(n-2) r^{4}}{r^{2} s^{2}}
\end{aligned}
$$


which then gives us

$$
|A|^{2}-\frac{1}{n-2} H^{2}-4=\frac{2(n-2)}{(n-1)} \frac{s^{2}}{r^{2}} .
$$

Hence for any $\varepsilon$ sufficiently small there exists a manifold of the form $\mathbb{S}^{2} \times \mathbb{S}^{n-2}$ such that

$$
|A|^{2}-\frac{1}{n-2} H^{2}-4=\varepsilon .
$$

Hence this shows that the quadratic bound in the connected sum theorem is the best that we can achieve.

3.3. Convexity and quadratic bounds. Here we investigate the relation of the quadratic bounds and curvature.

Lemma 3.2. We have the following equations:

$$
|A|^{2}-\frac{1}{n-1} H^{2}=-2 \lambda_{1} \lambda_{2}+\left(\lambda_{1}+\lambda_{2}-\frac{H}{n-1}\right)^{2}+\sum_{l=3}^{n}\left(\lambda_{l}-\frac{H}{n-1}\right)^{2}
$$

and

$$
\begin{aligned}
|A|^{2}-\frac{1}{n-2} H^{2} & =-2\left(\lambda_{1} \lambda_{2}+\lambda_{1} \lambda_{3}+\lambda_{2} \lambda_{3}\right)+\left(\lambda_{1}+\lambda_{2}+\lambda_{3}-\frac{H}{n-2}\right)^{2} \\
& +\sum_{l=4}^{n}\left(\lambda_{l}-\frac{H}{n-2}\right)^{2}
\end{aligned}
$$

This shows us that if $|A|^{2}-\frac{1}{n-1} H^{2} \leq 0$, then $\lambda_{1} \lambda_{2} \geq 0$ or $\lambda_{1} \lambda_{2} \leq 0$ for principal curvatures. If in addition we have mean convexity, that is, $H>0$, then there is a positive principal curvature which shows that all other principal curvatures are also non-negative. That is a quadratic bound and mean convexity implies convexity. For submanifolds of the sphere, if we have

$$
|A|^{2}-\frac{1}{n-1} H^{2}-2 K \leq 0
$$

then this does not imply convexity. Instead we have that for the sectional curvature

$$
2 K_{\mathcal{M}}\left(e_{1}, e_{2}\right)=2 \lambda_{1} \lambda_{2}+2 K \geq 2 K+\frac{1}{n-1} H^{2}-|A|^{2} \geq 0 .
$$

That is, the quadratic bound implies non-negative sectional curvature but not convexity. Similarly, the quadratic bound

$$
|A|^{2}-\frac{1}{n-2} H^{2} \leq 0, H \geq 0
$$

implies two-convexity in the Euclidean case but not in the spherical case.

\section{SElF-Shrinkers AND type $I$ SINGUlarities}

In this section we consider self-shrinkers which arise from type $I$ singularities. We shall show that, like the case of mean curvature flow in $\mathbb{R}^{n}$, a rescaled solution converges to a self-shrinker in $\mathbb{R}^{n}$. A proof for the similar case of Yang-Mills flow is given by [12] and the harmonic map heat flow case is well known. 
Definition 4.1. We say that our surface develops a singularity of type $I$ or a fast singularity as $t \rightarrow T$ if there exists a constant $C>0$ such that

$$
\sup _{\mathcal{M}_{t}}|A|^{2} \leq \frac{C}{T-t}
$$

Otherwise the singularity is of type $I I$.

Let us consider the case of a type $I$ singularity where $\max _{\mathcal{M}_{t}}|A|^{2} \leq \frac{1}{2(T-t)}$. By standard arguments we see that we can take a limit which converges to a selfshrinker, that is, a solution to the mean curvature flow that shrinks proportionally to its life time,

$$
X(t, x)=\sqrt{T-t} X_{0}(x) .
$$

Alternatively, it satisfies the following condition:

$$
H=\langle X, \nu\rangle \text {. }
$$

Theorem 4.2. If $\mathcal{M}^{n}, n \geq 2$ is compact such that $|A|^{2} \leq \alpha H^{2}, \alpha<1$ and satisfies $H=\langle X, \nu\rangle$, then $\mathcal{M}^{n}$ is isometric to a sphere of radius $R=\sqrt{n}$.

Proof. By differentiating the equation, we get that

$$
\begin{aligned}
\nabla_{i} H & =\left\langle X, e_{l}\right\rangle h_{l i}, \\
\nabla_{i} \nabla_{l} H & =h_{i j}-H h_{i l} h_{l j}+\left\langle X, e_{l}\right\rangle \nabla_{l} h_{i j} .
\end{aligned}
$$

Furthermore we get the equations,

$$
\begin{aligned}
\nabla_{l} H & =H-H|A|^{2}+\left\langle X, e_{l}\right\rangle \nabla_{l} H, \\
h_{i j} \nabla_{i} \nabla_{j} H & =|A|^{2}-H \operatorname{tr}\left(A^{3}\right)+\left\langle X, e_{l}\right\rangle \nabla_{l} h_{i j} \cdot h_{i j} .
\end{aligned}
$$

Simon's identity gives us the equation

$$
\begin{aligned}
& \triangle|A|^{2}=2 h_{i j} \nabla_{i} \nabla_{j} H+2|\nabla A|^{2}+2 \operatorname{tr}\left(A^{3}\right)-2|A|^{4}, \\
& \triangle|A|^{2}=2|\nabla A|^{2}+2|A|^{2}-2|A|^{4}+2\left\langle X, e_{l}\right\rangle \nabla_{l} h_{i j} h_{i j} .
\end{aligned}
$$

To prove this we consider the equation for $f=|A|^{2}-\alpha H^{2}$. First consider the case where $H \neq 0$. We will show that $f$ is superharmonic at a zero maximum

$$
\begin{aligned}
\triangle\left(|A|^{2}-\alpha H^{2}\right) & =\triangle|A|^{2}-\alpha 2 H \triangle H \\
& =2|\nabla A|^{2}-2 \alpha|\nabla H|^{2}+2 f\left(1-|A|^{2}\right)+\left\langle X, e_{l}\right\rangle \nabla_{l} f .
\end{aligned}
$$

We assume that $H \neq 0$, so that

$$
\begin{aligned}
2|\nabla A|^{2}-2 \alpha|\nabla H|^{2} & =2|\nabla A|^{2}-2 \frac{|A|^{2}-f}{H^{2}}|\nabla H|^{2} \\
& =\frac{2}{H^{2}}\left(H^{2}|\nabla A|^{2}+|A|^{2}\left(\left.\nabla H\right|^{2}\right)+2 \frac{f}{H^{2}}|\nabla H|^{2}\right. \\
& =\frac{2}{H^{2}}\left(H^{2}|\nabla A|^{2}+|A|^{2}|\nabla H|^{2}-4 \frac{|A|^{2}}{H^{2}}|\nabla H|^{2}+\frac{2 f}{H^{2}}|\nabla H|^{2}\right. \\
& =\frac{2}{H^{2}}\left(H^{2}|\nabla A|^{2}-H \nabla_{i}|A|^{2} \nabla_{i} H+|A|^{2}|\nabla H|^{2}\right) \\
& -4 \frac{|A|^{2}}{H^{2}}|\nabla H|^{2}+\frac{2}{H} \nabla_{i}|A|^{2} \nabla_{i} H+\frac{2 f}{H^{2}}|\nabla H|^{2} \\
& =\frac{2}{H^{2}}\left|h_{i j} \nabla_{l} H-\nabla_{l} h_{i j} H\right|^{2}-\frac{2 f}{H^{2}}|\nabla H|^{2}+\frac{4}{H} \nabla_{i} H \nabla_{i} f .
\end{aligned}
$$


Hence if $H \neq 0$ we have that

$$
\begin{aligned}
\triangle\left(|A|^{2}-\alpha H^{2}\right) & =\frac{2}{H^{2}}\left|h_{i j} \nabla_{l} H-\nabla_{l} h_{i j} H\right|^{2} \\
& -\frac{2 f}{H^{2}}|\nabla H|^{2}+\frac{4}{H} \nabla_{i} H \nabla_{i} f+2 f\left(1-|A|^{2}\right)+\left\langle X, e_{l}\right\rangle \nabla_{l} f .
\end{aligned}
$$

Suppose that $H=0$ and the inequality $|A|^{2} \leq \alpha H^{2} \Longrightarrow h_{i j}=0$ so that we also have that

$$
\nabla_{i} H=\left\langle X, e_{l}\right\rangle h_{l i}=0
$$

This then gives us

$$
\triangle f=2|\nabla A|^{2},
$$

and we can apply the maximum principle and conclude that

$$
f=|A|^{2}-\alpha H^{2} \equiv 0 .
$$

So that where $H \neq 0$,

$$
\left|h_{i j} \nabla_{i} H-\nabla_{l} h_{i j} H\right|^{2}=0
$$

and if $H=0$, again as $\nabla H=0$, we have

$$
\left|h_{i j} \nabla_{i} H-\nabla_{l} h_{i j} H\right|^{2}=0 .
$$

Splitting the tensor $h_{i j} \nabla_{l} H-\nabla_{l} h_{i j} H$ into symmetric and anti-symmetric parts, from Codazzi's equation we have

$$
\left|h_{i j} \nabla_{l} H-h_{i l} \nabla j H\right|^{2}=0 .
$$

Then consider an orthonormal frame $\left\{e_{1}, \ldots, e_{m}\right\}$ so that $e_{1}=\frac{\nabla H}{|\nabla H|}$ so that

$$
0=\left|h_{i j} \nabla_{l} H-h_{i l} \nabla_{j} H\right|^{2}=2|\nabla H|^{2}\left(|A|^{2}-\sum_{i=1}^{n} h_{1 l}^{2}\right),
$$

so that either $|\nabla H|^{2}=0$ or that $|A|^{2}=\sum_{i=1}^{n} h_{1 i}^{2}$. If we have that $|\nabla H|^{2}=0$, this then implies that $|\nabla A|^{2}=0$. Then we have a sphere 9 and we are done. Otherwise assume that there exists a point $x \in \mathcal{M}$ such that $|A|^{2}=\sum_{i=1}^{n} h_{1 i}^{2}$ so that

$$
|A|^{2}=h_{11}^{2}+2 \sum_{i=2}^{n} h_{1 i}^{2}+\sum_{i \neq j}^{n} h_{i j}^{2} .
$$

This can only occur if $h_{i j}=0$ for $i \neq j \neq 1$, hence $|A|^{2}=h_{11}^{2}=H^{2}$. Hence this holds on the entire manifold. But $|A|^{2}=\alpha H^{2}, \alpha<1$, which is a contradiction, hence we must have $|\nabla H|^{2}=0$ and $\mathcal{M}=\mathbb{S}^{n}$ isometrically.

By modifying the argument of [6], we obtain the following classification,

Theorem 4.3. Let $\tilde{\mathcal{M}}_{\infty}$ be a smooth limiting solution in $\mathbb{R}^{n+1}$ satisfying the selfshrinker equation such that $|A|^{2} \leq \alpha H^{2}, \alpha \leq 1$. Then $\tilde{\mathcal{M}}_{\infty}$ is isometric to one of the following:
(1) $\mathbb{S}^{n}$
(2) $\mathbb{S}^{n-m} \times \mathbb{R}^{m}$,
(3) $\Gamma \times \mathbb{R}^{n-1}$.

where $\Gamma$ is one of the homothetically shrinking curves found by Abresch and Langer. 


\section{Huisken-Sinestrari estimates and type $I I$ Singularities}

In this section, we survey the Huisken-Sinestrari estimates for surfaces of nonnegative mean curvature. These estimates show that as we approach a singular time, the curvature of the submanifold becomes weakly convex. Although the statement and proofs below are stated for $\mathbb{S}^{n}$, as noted in the paper [7] the proofs can be generalised to Riemannian manifolds. In our case, we have a symmetric space with positive curvature so the proofs in [7] extend almost trivially.

\subsection{Convexity estimates.}

Theorem 5.1 ([7]). Suppose that $X_{0}: \mathcal{M}^{n} \rightarrow \mathbb{S}^{n+1}$ is a smooth closed hypersurface immersion with non-negative mean curvature. For each $k, 2 \leq k \leq n$, and any $\eta>0$ there is a constant $C_{\eta, k}$ depending on $n, k, \eta$ and the initial data, such that everywhere on $\mathcal{M} \times[0, T]$ we have the estimate for the $k$-th symmetric polynomial of the principal curvatures

$$
S_{k} \geq-\eta H^{k}-C_{\eta, k}
$$

The arbitrariness of $\eta$ breaks the scaling invariance in inequality (5.1). This implies that near a singularity where $S_{1}=H$ becomes unbounded, each $S_{k}$ becomes non-negative after rescaling. Hence we have the following corollary:

Corollary 5.2. Let $\mathcal{M}_{t}$ be a mean convex solution of mean curvature on the maximal tie interval $[0, T)$ as in the previous theorem. Then any smooth rescaling of the singularity for $t \rightarrow T$ is convex.

Applying the above theorem we get the flowing classification of type $I I$ singularities.

Theorem 5.3. If $\mathcal{M}_{0}$ has non-negative mean curvature, then any limiting flow of a type II singularity has convex surfaces $\tilde{\mathcal{M}}_{\tau}, \tau \in \mathbb{R}$. Furthermore, $\tilde{\mathcal{M}}_{\tau}$ is a strictly convex translating soliton of (up to rigid motions) $\tilde{\mathcal{M}}_{\tau}=\mathbb{R}^{n-k} \times \Sigma_{\tau}^{k}$ where $\Sigma_{\tau}^{k}$ is a lower-dimensional strictly convex translating soliton in $\mathbb{R}^{k+1}$.

\section{Cylindrical estimates}

In this section, we prove cylindrical estimates. That is, we show that at a singular point if the submanifold does not become spherical, then it becomes cylindrical.

6.1. Convexity estimates and Simons identity. We will use the following consequence of the Huisken-Sinestrari convexity estimates. Note that although the theorems in the paper are stated for Euclidean space ([7]), they can be easily generalised to a symmetric space or even a general Riemannian manifold.

Lemma 6.1. Suppose that $X_{0}: \mathcal{M}^{n} \rightarrow \mathbb{S}^{n+1}$ is a smooth closed hypersurface with non-negative mean curvature. Then for the solution of the mean curvature flow $\mathcal{M}_{t}$, for any $\eta>0$ there exists $C_{\eta}=C_{\eta}(n, \alpha)$ such that

$$
\lambda_{1} \geq-\eta H-C_{\eta} \text {. }
$$

To apply the iteration argument to get our estimates, we will need to bound the non-linearity appearing in Simons identity,

$$
\triangle|A|^{2}=2\left\langle h_{i j}, \nabla_{i} \nabla_{j} H\right\rangle+2|\nabla A|^{2}+2 Z,
$$


where $Z=H \operatorname{tr}\left(A^{3}\right)-|A|^{4}+n K\left(|A|^{2}-\frac{1}{n} H^{2}\right)$. In this case, we will not be able to obtain a positive lower bound. However combining the quadratic estimate and the convexity estimate, we can show that the negative part of the non-linearity is of lower order. This will allow us to push through the iteration argument.

Lemma 6.2. Assuming the mean convex quadratic bound (1.1), we have the following estimate on $Z$. There exists a constant $\gamma_{1}$ depending only on $n, \alpha_{0}$, such that for any $\eta>0$ there exists $K_{\eta}$ such that

$$
Z \geq \gamma_{1} H^{2}\left(|A|^{2}-\frac{1}{n-1} H^{2}-\eta H^{2}\right)-K_{\eta}\left(H^{3}+H^{2}+H+1\right) .
$$

Proof. First note the equation,

$$
|A|^{2}-\frac{1}{n-1} H^{2}=\frac{1}{n-1}\left(\sum_{1<i<j}\left(\lambda_{i}-\lambda_{j}\right)^{2}+\lambda_{1}\left(n \lambda_{1}-2 H\right)\right) .
$$

For the proof, we require a bound on the two-convexity from below. First we give a simple estimate if $n=4$. By the quadratic estimate $|A|^{2}-\frac{1}{n-2} H^{2}-4 K \leq 0$, and let us assume that $\lambda_{1} \leq \lambda_{2} \leq \lambda_{3} \leq \lambda_{4}$; then

$$
\left(\lambda_{1}+\lambda_{2}\right)\left(\lambda_{2}+\lambda_{4}\right)+4 K \geq 0 .
$$

Now we have that

$$
2\left(\lambda_{4}+\lambda_{2}\right) \geq \lambda_{4}+\lambda_{2}+\lambda_{3}+\lambda_{1} \geq C .
$$

Rearranging this gives us

$$
-\frac{1}{\lambda_{2}+\lambda_{4}} \geq-\frac{2}{C}
$$

This leads to the following estimate on the two-convexity:

$$
\lambda_{1}+\lambda_{2} \geq-\frac{2 K}{C}
$$

and by (3.2)

$$
2 \lambda_{i} \lambda_{j}+2 \lambda_{i} \lambda_{k}+2 \lambda_{j} \lambda_{k}+4 K \geq 4 K++\frac{1}{n-2} H^{2}-|A|^{2} \geq \gamma H^{2},
$$

where $H \geq C$. In general we can just use the fact that $\lambda_{1}+\lambda_{2} \geq-\eta H+C_{\eta}$. Now $Z$ may be written as

$$
Z=\sum_{j=2}^{n}\left(\lambda_{1} \lambda_{j}+K\right)\left(\lambda_{1}-\lambda_{j}\right)^{2}+\sum_{1<i<j}\left(\lambda_{i} \lambda_{j}+K\right)\left(\lambda_{i}-\lambda_{j}\right)^{2} .
$$

The second term may be estimated as follows:

$$
\begin{aligned}
\sum_{1<i<j}\left(\lambda_{i} \lambda_{j}+K\right)\left(\lambda_{i}-\lambda_{j}\right)^{2} & =\sum_{1<i<j}\left(\lambda_{i} \lambda_{j}+\lambda_{i} \lambda_{k}+\lambda_{j} \lambda_{k}+2 K\right)\left(\lambda_{i}-\lambda_{j}\right)^{2} \\
& -\sum_{1<i<j}\left(\lambda_{k}\left(\lambda_{i}+\lambda_{j}\right)+K\right)\left(\lambda_{i}-\lambda_{j}\right)^{2} .
\end{aligned}
$$


Now we choose $\lambda_{k}=\lambda_{1}<0$. Otherwise if $\lambda_{1}>0$ the second fundamental form is convex and this estimate has been proven for convex surfaces in 4]. Hence we have the estimate

$$
\begin{aligned}
-2 & \sum_{1<i<j}\left(\lambda_{k}\left(\lambda_{i}+\lambda_{j}\right)+K\right)\left(\lambda_{i}-\lambda_{j}\right)^{2} \\
& \geq-C \lambda_{1}\left(\lambda_{1}+\lambda_{2}\right) \sum_{1<i<j}\left(\lambda_{i}-\lambda_{j}\right)^{2}-C K \sum_{1<i<j}\left(\lambda_{i}-\lambda_{j}\right)^{2} \\
& \geq \lambda_{1} C_{1} K\left(\frac{1}{n-2} H^{2}+4 K\right)-C_{2} K\left(\frac{1}{n-2} H^{2}+4 K\right) .
\end{aligned}
$$

The other term is then

$$
\begin{aligned}
& \sum_{1<i<j}\left(\lambda_{i} \lambda_{j}+\lambda_{i} \lambda_{1}+\lambda_{1} \lambda_{j}+2 K\right)\left(\lambda_{i}-\lambda_{j}\right)^{2} \\
& \quad \geq\left(4 K+\frac{1}{n-2} H^{2}-|A|^{2}\right) \sum_{1<i<j}\left(\lambda_{i}-\lambda_{j}\right)^{2} \\
& \quad \geq\left(4 K+\frac{1}{n-2} H^{2}-|A|^{2}\right)\left[(n-1)\left(\frac{1}{n-1} H^{2}-|A|^{2}\right)-\lambda_{1}\left(n \lambda_{1}-2 H\right)\right] .
\end{aligned}
$$

We note that we have the estimate

$$
\begin{aligned}
\lambda_{1}^{2}, \lambda_{n}^{2} \leq|A|^{2} & \leq \frac{1}{n-2}|H|^{2}+4 K \\
& \leq\left(\frac{1}{\sqrt{n-2}} H+2 \sqrt{K}\right)^{2}
\end{aligned}
$$

and $\lambda_{n} \geq 0, H \geq 0$. We have the estimate

$$
\lambda_{n} \leq \frac{1}{\sqrt{n-2}} H+2 \sqrt{K} .
$$

And similarly as $\lambda_{1} \leq 0, H \geq 0$ we have

$$
-\lambda_{1} \leq \frac{1}{\sqrt{n-2}} H+2 \sqrt{K}
$$

We collect the remaining terms with a coefficient of $\lambda_{1}$, and get

$$
\lambda_{1}\left(\sum_{j=2} \lambda_{j}\left(\lambda_{1}-\lambda_{j}\right)^{2}+\left(4 K+\frac{1}{n-2} H^{2}-|A|^{2}\right)\left(2 H-n \lambda_{1}\right)\right) .
$$

Here we use the estimate $\lambda_{1} \geq-\eta H-C_{\eta}$ to estimate the first factor. To estimate the second factor in the brackets, we have

$$
\begin{aligned}
& \sum_{j=2} \lambda_{j}\left(\lambda_{1}-\lambda_{j}\right)^{2}+\gamma H^{2}\left(2 H-n \lambda_{1}\right) \\
& \quad \leq C H\left(\frac{1}{n-1} H^{2}+4 K\right)+\gamma H^{2}\left(2 H+\frac{1}{\sqrt{n-2}} H+2 \sqrt{K}\right) \\
& \quad \leq C_{1} H^{3}+C_{2} H^{2}+C_{3} H,
\end{aligned}
$$

as $\left(\lambda_{1}-\lambda_{j}\right)^{2} \leq C|A|^{2}, \lambda_{j} \leq \lambda_{n} \leq \frac{1}{\sqrt{n-2}} H+2 \sqrt{K},-\lambda_{1} \leq \frac{1}{\sqrt{n-2}} H+2 \sqrt{K}$. 
6.2. Cylindrical estimates. In this subsection, we derive cylindrical estimates.

Theorem 6.3 (Cylindrical estimates, 8]). Let $\mathcal{M}_{t}$ be a smooth solution of mean curvature flow with $n \geq 4$ and initial data satisfying $|A|^{2}<\frac{1}{n-2} H^{2}+4 K, H>0$. Then, for any $\eta>0$ there exists a constant $C_{\eta}=C_{\eta}(n)$ such that

$$
|A|^{2}-\frac{1}{n-1} H^{2} \leq \eta H^{2}+C_{\eta}
$$

on $\mathcal{M}_{t}$ for any $t \in(0, T[$.

The above theorem gives the simple corollary that

Corollary 6.4. Let $\mathcal{M}_{t}$ be a smooth solution of mean curvature flow with $n \geq 4$ and initial data satisfying $|A|^{2}<\frac{1}{n-2} H^{2}+4 K, H>0$. Then, for any $\eta>0$ there exists constants $c=c(n), C_{\eta}=C_{\eta}(n)$ such that

$$
\left|\lambda_{1}\right| \leq \eta H \Longrightarrow\left(\lambda_{i}-\lambda_{j}\right)^{2} \leq c \eta H^{2}+C_{\eta} .
$$

Proof. Let $\mathcal{M}_{t}$ be a smooth solution of mean curvature flow with $n \geq 3$ and initial data satisfying $|A|^{2}<\frac{1}{n-2} H^{2}+4 K$. As above we have the estimate

$$
|A|^{2}-\frac{1}{n-1} H^{2}=\frac{1}{n-1}\left(\sum_{1<i<j}\left(\lambda_{i}-\lambda_{j}\right)^{2}+\lambda_{1}\left(n \lambda_{1}-2 H\right)\right) .
$$

Then, by Theorem 6.3 for any $\eta>0$ there exists a constant $C_{\eta}=C_{\eta}(n)$ such that

$$
|A|^{2}-\frac{1}{n-1} H^{2} \leq \eta H^{2}+C_{\eta} .
$$

The bound $\left|\lambda_{1}\right| \leq \eta H$ then gives the desired estimate.

To derive cylindrical estimates we will consider the following function:

$$
f_{\sigma, \eta}=\frac{|A|^{2}-\left(\frac{1}{n-1}+\eta\right) H^{2}}{\left(a H^{2}+b\right)^{1-\sigma}}=\frac{|A|^{2}-\left(\frac{1}{n-1}+\eta\right) H^{2}}{W^{1-\sigma}}
$$

where we let $a=\alpha_{n}-\left(\frac{1}{n-1}+\eta\right)+\frac{\varepsilon}{2 n(n-1)}, b=\beta_{n} K$.

Lemma 6.5. For all $0 \leq \sigma \leq \sigma_{1}$ where $\sigma_{1}$ only depends on $\mathcal{M}_{0}$ we have the inequality

$$
\begin{aligned}
\frac{\partial}{\partial t} f_{\sigma, \eta} \leq & \triangle f_{\sigma, \eta}+\frac{4 a(1-\sigma) H}{W}\left\langle\nabla f_{\sigma, \eta}, \nabla H\right\rangle-\frac{\varepsilon}{12 W^{1-\sigma}}|\nabla H|^{2}-n \varepsilon K f_{\sigma, \eta} \\
& +2 \sigma\left(|A|^{2}+n K\right) f_{\sigma, \eta} .
\end{aligned}
$$

Proof. As a first step we get an evolution equation for $f_{0}=f_{0, \eta}$,

$$
\begin{aligned}
\frac{\partial}{\partial t} f_{0}= & \frac{1}{a H^{2}+b}\left(\triangle\left(|A|^{2}-\left(\frac{1}{n-1}+\eta\right) H^{2}\right)-f_{0} \triangle\left(a H^{2}\right)\right. \\
& \left.-2\left(|\nabla A|^{2}-\left(\frac{1}{n-1}+\eta+a f_{0}\right)|\nabla H|^{2}\right)\right) \\
+ & 2 f_{0} \frac{1}{a H^{2}+b}\left(b|A|^{2}-2 a n K H^{2}-b n K\right) .
\end{aligned}
$$


Furthermore we have that

$$
\begin{aligned}
\nabla_{i} f_{0}= & \frac{1}{a H^{2}+b}\left(\nabla_{i}\left(|A|^{2}-\left(\frac{1}{n-1}+\eta\right) H^{2}\right)-f_{0} \nabla_{i}\left(a H^{2}\right)\right), \\
\triangle f_{0}= & \frac{1}{a H^{2}+b}\left(\triangle\left(|A|^{2}-\left(\frac{1}{n-1}+\eta\right) H^{2}\right)-f_{0} \triangle\left(a H^{2}\right)\right) \\
& -4 \frac{a H}{a H^{2}+b}\left\langle\nabla_{i} H, \nabla_{i} f_{0}\right\rangle .
\end{aligned}
$$

Note that we chose $a, b$ so that $f_{0} \leq 1$. Hence we get that

$$
\begin{aligned}
\frac{\partial}{\partial t} f_{0} \leq & \triangle f_{0}+4 \frac{a H}{a H^{2}+b}\left\langle\nabla_{i} f_{0}, \nabla_{i} H\right\rangle \\
& -\frac{2}{a H^{2}+b}\left(|\nabla A|^{2}-\left(a+\left(\frac{1}{n-1}+\eta\right)\right)|\nabla H|^{2}\right) \\
& +2 f_{0} \frac{1}{a H^{2}+b}\left(b|A|^{2}-2 a n K H^{2}-b n K\right)-\frac{4}{W}\left(\frac{1}{n-1}+\eta n\right) K H^{2} .
\end{aligned}
$$

Now we use the fact that $a=\alpha_{n}-\left(\frac{1}{n-1}+\eta\right)+\frac{\varepsilon}{2 n(n-1)}, b=\beta_{n} K$, to get that

$$
\begin{aligned}
& \left(b|A|^{2}-2 a n K H^{2}-b n K\right)=\beta_{n} K\left(|A|^{2}-2 a \frac{n}{\beta_{n}} H^{2}-n K\right) \\
& \leq \beta_{n} K\left(\alpha_{n} H^{2}+\left(\beta_{n}-n\right) K-\frac{2 n}{\beta_{n}}\left(\alpha_{n}-\frac{1}{n}+\frac{1}{n}-\frac{1}{n-1}-\eta+\frac{\varepsilon}{2 n(n-1)}\right) H^{2}\right) \\
& \leq \beta_{n} K\left(\left(\beta_{n}-n\right) K-\frac{\varepsilon}{(n-1) \beta_{n}} H^{2}\right)-2 n K\left(\frac{1}{n}-\frac{1}{n-1}-\eta\right) H^{2} .
\end{aligned}
$$

Since $\alpha_{n}-\frac{2 n}{\beta_{n}}\left(\alpha_{n}-\frac{1}{n}\right)=0$ we have that for all $n \geq 4$

$$
-K\left(\frac{\varepsilon}{n-1} H^{2}+n \varepsilon \beta_{n} K\right) \leq-\frac{n \varepsilon}{2} K\left(a H^{2}+b\right)
$$

and using the fact that $f_{0} \leq 1$ the second term gives us

$$
\frac{4 n K f_{0}}{W}\left(-\frac{1}{n}+\frac{1}{n-1}+\eta\right) H^{2}-\frac{4}{W}\left(\frac{1}{n-1}+\eta n\right) K H^{2} \leq 0 .
$$

We have that for $n \geq 3, \frac{3}{n+2}-\left(a+\left(\frac{1}{n-1}+\eta\right)\right) \geq \frac{\varepsilon}{16}$ we get the estimate

$$
\frac{\partial}{\partial t} f_{0} \leq \triangle f_{0}+\frac{4 a H}{a H^{2}+b}\left\langle\nabla H, \nabla f_{0}\right\rangle-\frac{\varepsilon}{8\left(a H^{2}+b\right)}|\nabla H|^{2}-n \varepsilon K f_{0} .
$$

Note that we have a negative coefficient in front of the $|\nabla H|^{2}$ term. This will allow us to use the iteration method, without using a bound on $\left|h_{j k} \nabla_{i} H-H \nabla_{i} h_{j k}\right|^{2}$. Now we compute the evolution of the term $W^{\sigma}=\left(a H^{2}+b\right)^{\sigma}$. First we have

$$
\begin{aligned}
\triangle W^{\sigma} & =\sigma \nabla^{i}\left(W^{\sigma-1} 2 a H \nabla_{i} H\right) \\
& =2 \sigma a H W^{\sigma-1} \triangle H+4 \sigma(\sigma-1) a^{2} H^{2} W^{\sigma-2}|\nabla H|^{2}+2 a \sigma W^{\sigma-2}|\nabla H|^{2} .
\end{aligned}
$$

And hence we get the evolution equation

$$
\begin{aligned}
\partial_{t} W^{\sigma}= & \triangle W^{\sigma}-4 \sigma(\sigma-1) a^{2} H^{2} W^{\sigma-2}|\nabla H|^{2}-2 a \sigma W^{\sigma-1}|\nabla H|^{2} \\
& +2 \sigma a H^{2} W^{\sigma-1}\left(|A|^{2}+n K\right) .
\end{aligned}
$$


This gives us the evolution equation

$$
\begin{aligned}
\partial_{t} f_{\sigma, \eta}= & \partial_{t}\left(f_{0} W^{\sigma}\right) \leq \triangle f_{\sigma, \eta}-2\left\langle\nabla_{i} f_{0}, \nabla_{i} W^{\sigma}\right\rangle \\
& +4 a H W^{\sigma-1}\left\langle\nabla_{i} f_{0}, \nabla_{i} H\right\rangle-\frac{\varepsilon}{12} W^{\sigma-1}|\nabla H|^{2} \\
& -n \varepsilon K f_{0} W^{\sigma}-4 \sigma(\sigma-1) a^{2} H^{2} W^{\sigma-2} f_{0}|\nabla H|^{2}-2 a \sigma W^{\sigma-1} f_{0}|\nabla H|^{2} \\
& +2 a \sigma H^{2} W^{\sigma-1}\left(|A|^{2}+n K\right) .
\end{aligned}
$$

The coefficient of $|\nabla H|^{2}$ is given by

$$
-4 \sigma(\sigma-1) a^{2} H^{2} \frac{f_{0}}{W^{2-\sigma}}-2 a \sigma \frac{f_{0}}{W^{1-\sigma}}=-2 \frac{f_{\sigma, \eta}}{W}\left(a \sigma+2 a^{2} \sigma(1-\sigma) \frac{H^{2}}{W}\right)
$$

which is negative if $\sigma \in[0,1]$. This then proves the lemma.

Using the above evolution inequality, we can derive the evolution inequality for the $L^{p}$ norm of $f_{+}^{p}$.

Lemma 6.6. For $\sigma, \eta \in(0,1)$ we have the following evolution equation:

$$
\begin{aligned}
\partial_{t} \int_{\mathcal{M}_{t}} f_{+}^{p} & +p(p-1) \int_{\mathcal{M}_{t}} f_{+}^{p-2}\left|\nabla f_{+}\right|^{2}+\frac{\varepsilon}{12} p \int_{\mathcal{M}_{t}} W^{\sigma-1}|\nabla H|^{2} f_{+}^{p-1} \\
\leq & 4 p a(1-\sigma) \int_{\mathcal{M}_{t}} \frac{H}{W}|\nabla H|\left|\nabla f_{+}\right| f_{+}^{p-1} d \mu \\
& +\int\left(2 \sigma p\left(|A|^{2}+n K\right)-n K \varepsilon\right) f_{+}^{p},
\end{aligned}
$$

for some $p \geq c_{2}$.

Proof. We have the evolution equation

$$
\partial_{t} \int f_{+}^{p} d \mu=p \int\left(f_{+}^{p-1} \partial_{t} f_{+}-H^{2} f_{+}^{p}\right) d \mu .
$$

Using the evolution equation above for $f_{\sigma, \eta}$ we have that

$$
\begin{aligned}
\frac{\partial}{\partial t} f_{\sigma, \eta} \leq & \triangle f_{\sigma, \eta}+\frac{4 a(1-\sigma) H}{W}\left\langle\nabla f_{\sigma, \eta}, \nabla H\right\rangle-\frac{\varepsilon}{12 W^{1-\sigma}}|\nabla H|^{2}-n \varepsilon K f_{\sigma, \eta} \\
& +2 \sigma\left(|A|^{2}+n K\right) f_{\sigma, \eta} .
\end{aligned}
$$

We insert this into (6.2) to get

$$
\begin{aligned}
\partial_{t} \int f_{+}^{p} d \mu & \leq p(p-1) \int f_{+}^{p-2}\left|\nabla f_{+}\right|^{2}+\frac{\varepsilon}{12} p \int W^{\sigma-1}|\nabla H|^{2} f_{+}^{p-1} \\
& +5 p a(1-\sigma) \int \frac{H}{W}|\nabla H|\left|\nabla f_{+}\right| f_{+}^{p-1} \\
& +2 \sigma p \int\left(\left(|A|^{2}+n K\right) f_{+}^{p}-n K \varepsilon f_{+}^{p}\right) d \mu .
\end{aligned}
$$

In order to use the good negative gradient type terms, we need a Poincaré type inequality that we obtain by integrating the Simon's identity together with the lower bound on the $Z$ term. In the following, we let

$$
h_{i j}^{\eta}=h_{i j}-\left(\frac{1}{n-1}+\eta\right) g_{i j} .
$$


Proposition 6.7 (Poincaré type inequality). There exists $c_{3}=c_{3}\left(\mathcal{M}_{0}\right)$ and $C=$ $C(n, \eta)$ such that

$\frac{2}{\eta c_{3}} \int_{\mathcal{M}_{t}} W f_{+}^{p} \leq \frac{(p+1)}{\beta} \int_{\mathcal{M}_{t}} f_{+}^{p-2}|\nabla f|^{2}+(1+\beta p) \int \frac{f_{+}^{p}}{W}|\nabla H|^{2}+C \int_{\mathcal{M}_{t}} \frac{H^{3}}{W}|\nabla H|^{2}$ for $\beta>0, n p>2$.

Proof. By Simon's identity

$$
\begin{aligned}
\triangle|A|^{2} & =2\left\langle h_{i j} \nabla_{i} \nabla_{j} H\right\rangle+2|\nabla A|^{2}+2\left(H \operatorname{tr}\left(A^{3}\right)-|A|^{4}\right)+2 n K \sum_{i<j}\left(\lambda_{i}-\lambda_{j}\right)^{2} \\
& =2\left\langle h_{i j}, \nabla_{i} \nabla_{j} H\right\rangle+2|\nabla A|^{2}+2 Z .
\end{aligned}
$$

Now we wish to compute

$$
\triangle \frac{\left(|A|^{2}-\left(\frac{1}{n-1}+\eta\right) H^{2}\right.}{W} .
$$

And this gives us

$$
\begin{aligned}
\triangle f_{\sigma, \eta} & \geq 2 W^{\sigma-1}\left\langle h_{i j}-\left(\frac{1}{n-1}+\eta\right) g_{i j}, \nabla_{i} \nabla_{j} H\right\rangle+2 Z W^{\sigma-1} \\
& -2(1-\sigma) \frac{H f_{\sigma, \eta}}{W} \triangle H-4 a(1-\sigma) \frac{H}{W}\left\langle\nabla_{i} H, \nabla_{i} f_{\sigma, \eta}\right\rangle .
\end{aligned}
$$

We multiply by $\frac{f_{\sigma, \eta}^{p}}{W^{\sigma}}$ and integrate to get

$$
\int \frac{f_{\sigma, \eta}^{p} \Delta f_{\sigma, \eta}}{W^{\sigma}}=-p \int \frac{f_{\sigma, \eta}^{p-1}\left|\nabla f_{\sigma}\right|^{2}}{W^{\sigma}}+\sigma \int \frac{f_{\sigma, \eta}^{p}\left\langle\nabla f_{\sigma, \eta}, \nabla W\right\rangle}{W^{\sigma+1}}
$$

and

$$
\nabla \frac{1}{W^{\sigma}}=-\sigma W^{-\sigma-1} \nabla W=-2 a \sigma H W^{-\sigma-1} \nabla H .
$$

Hence we get

$$
\begin{aligned}
\int \frac{f_{\sigma, \eta}^{p} \Delta f_{\sigma, \eta}}{W^{\sigma}} & \geq \int 2 \frac{f_{\sigma, \eta}^{p}\left\langle h_{i j}^{\eta}, \nabla_{i} \nabla_{j} H\right\rangle}{W}+\int \frac{2 Z f_{\sigma, \eta}^{p}}{W} \\
& -2 a(1-\sigma) \int \frac{f_{\sigma, \eta}^{p+1} H \triangle H}{W^{\sigma+1}}-4 a(1-\sigma) \int \frac{f_{\sigma, \eta}^{p} H\left\langle\nabla_{i} H, \nabla_{i} f_{\sigma, \eta}\right\rangle}{W^{1+\sigma}} .
\end{aligned}
$$

The first term of (6.3) gives us

$$
\begin{aligned}
\int 2 \frac{f_{\sigma, \eta}^{p}\left\langle h_{i j}^{\eta}, \nabla_{i} \nabla_{j} H\right\rangle}{W} & =-2 \int \frac{-2 a f_{\sigma, \eta}^{p} H}{W^{2}}\left\langle h_{i j}^{\eta}, \nabla_{i} H \nabla_{j} H\right\rangle \\
& -2 p \int \frac{f_{\sigma, \eta}^{p-1} H}{W}\left\langle h_{i j}^{\eta}, \nabla_{i} H \nabla_{j} f_{\sigma, \eta}\right\rangle-\int \frac{f_{\sigma, \eta}^{p}}{W}\left\langle\nabla_{i} h_{i j}^{\eta}, \nabla H\right\rangle \\
& =4 a \int \frac{f_{\sigma, \eta}^{p}\left\langle h_{i j}^{\eta}, \nabla_{i} H \nabla_{j} H\right\rangle}{W^{2}} \\
& -2 p \int \frac{f_{\sigma, \eta}^{p}}{W}\left\langle h_{i j}^{\eta}, \nabla_{i} H \nabla_{j} f_{\sigma, \eta}\right\rangle-2 \int \frac{f_{\sigma, \eta}^{p}}{W}|\nabla H|^{2} .
\end{aligned}
$$


The third term in (6.3) gives us

$$
\begin{aligned}
-2 a & (1-\sigma) \int \frac{H f_{\sigma, \eta}^{p+1} \triangle H}{W^{\sigma+1}} \\
& =2 a(1-\sigma) \int(p+1) \frac{H f_{\sigma, \eta}^{p}}{W^{\sigma+1}}\left\langle\nabla f_{\sigma, \eta}, \nabla H\right\rangle+\frac{f_{\sigma, \eta}^{p+1}|\nabla H|^{2}}{W^{\sigma+1}} \\
& -2 a(1-\sigma) \int(\sigma+1) \frac{2 a H^{2}}{W^{\sigma+1}}\left\langle\nabla f_{\sigma, \eta}, \nabla H\right\rangle \\
& =2 a(1-\sigma) \int\left((p+1) \frac{H f_{\sigma, \eta}^{p}}{W^{\sigma+1}}-\frac{2(1+\sigma) a H^{2}}{W^{1+\sigma}}\right)\left\langle\nabla f_{\sigma, \eta}, \nabla H\right\rangle \\
& +2 a(1-\sigma) \int \frac{f_{\sigma, \eta}^{p+1}}{W^{\sigma+1}}|\nabla H|^{2} .
\end{aligned}
$$

Hence (6.2) becomes,

$$
\begin{aligned}
\int \frac{f_{\sigma, \eta}^{p}}{W^{\sigma}} \triangle f_{\sigma, \eta} & \geq \int \frac{2 Z f_{\sigma, \eta}^{p}}{W} d \mu+\int \frac{4 a H f_{\sigma, \eta}^{p}}{W^{2}}\left\langle h_{i j}^{\eta}, \nabla_{j} H \nabla_{i} H\right\rangle \\
& -2 p \int \frac{H f_{\sigma, \eta}^{p-1}}{W}\left\langle h_{i j}^{\eta}, \nabla_{i} H \nabla_{j} f_{\sigma, \eta}\right\rangle-2 \int \frac{f_{\sigma, \eta}^{p}}{W}|\nabla H|^{2} \\
& +2 a(1-\sigma)(p+1) \int \frac{H f_{\sigma, \eta}^{p}}{W^{\sigma+1}}\left\langle\nabla f_{\sigma, \eta}, \nabla H\right\rangle \\
& -4 a^{2}\left(1-\sigma^{2}\right) \int \frac{H^{2} f_{\sigma, \eta}^{p}}{W^{\sigma+2}}\left\langle\nabla f_{\sigma, \eta}, \nabla H\right\rangle \\
& +2 a(1-\sigma) \int \frac{f_{\sigma, \eta}^{p+1}}{W^{\sigma+1}}|\nabla H|^{2}-4 a(1-\sigma) \int \frac{H f_{\sigma, \eta}^{p}}{W^{1+\sigma}}\left\langle\nabla H, \nabla f_{\sigma, \eta}\right\rangle .
\end{aligned}
$$

We now split the gradient terms into two types, $\frac{f_{\sigma, \eta}^{p}}{W}|\nabla H|^{2}$ and $\frac{f_{\sigma, \eta}^{p-1}}{\sqrt{W}}\left|\nabla f_{\sigma, \eta}\right||\nabla H|$ terms,

$$
\begin{aligned}
& -2 p \int \frac{H}{W} f_{\sigma, \eta}^{p-1}\left\langle h_{i j}^{\eta}, \nabla_{i} H \nabla_{j} f_{\sigma, \eta}\right\rangle+2 a(1-\sigma)(p+1) \int \frac{H f_{\sigma, \eta}^{p}}{W^{\sigma+1}}\left\langle\nabla H, \nabla f_{\sigma, \eta}\right\rangle \\
& -4 a^{2}\left(1-\sigma^{2}\right) \int \frac{f_{\sigma, \eta}^{p} H^{2}}{W^{\sigma+2}}\left\langle\nabla f_{\sigma, \eta}, \nabla H\right\rangle-4 a(1-\sigma) \int \frac{H f_{\sigma, \eta}^{p}}{W^{1+\sigma}}\left\langle\nabla H, \nabla f_{\sigma, \eta}\right\rangle \\
& \leq C p \int \frac{f_{\sigma, \eta}^{p-1}}{\sqrt{W}}\left|\nabla f_{\sigma, \eta}\right||\nabla H|,
\end{aligned}
$$

where we used the facts that

$$
\frac{H}{\sqrt{W}} \leq C, \quad \frac{|A|}{\sqrt{W}} \leq C, \quad h_{i j}^{\eta}=h_{i j}-\left(\frac{1}{n-1}+\eta\right) g_{i j},
$$

which gives the estimate

$$
\left|\frac{\left\langle h_{i j}^{\eta}, \nabla_{i} H \nabla_{j} f_{\sigma, \eta}\right\rangle}{\sqrt{W}}\right| \leq C|\nabla H|\left|\nabla f_{\sigma, \eta}\right| .
$$


Next we have that

$$
\begin{aligned}
\int \frac{4 a H f_{\sigma, \eta}^{p}}{W^{2}}\left\langle h_{i j}^{\eta}, \nabla_{i} H \nabla_{j} H\right\rangle & -2 \int \frac{f_{\sigma, \eta}^{p}}{W}|\nabla H|^{2}+2 a(1-\sigma) \int \frac{f_{\sigma, \eta}^{p+1}}{W^{\sigma+1}}|\nabla H|^{2} \\
& \leq C \int \frac{f_{\sigma, \eta}^{p}}{W}|\nabla H|^{2} .
\end{aligned}
$$

This gives us the estimate

$$
\int \frac{2 Z}{W} f_{\sigma, \eta}^{p} \leq C p \int \frac{f_{\sigma, \eta}^{p-1}}{\sqrt{W}}|\nabla H|\left|\nabla f_{\sigma, \eta}\right|+C \int \frac{f_{\sigma, \eta}^{p}}{H^{2}}|\nabla H|^{2} .
$$

We use Young's inequality to get

$$
2 \frac{f_{\sigma, \eta}^{p-1}}{\sqrt{W}}|\nabla H|\left|\nabla f_{\sigma, \eta}\right| \leq \frac{f_{\sigma, \eta}^{p-2}}{\beta}\left|\nabla f_{\sigma, \eta}\right|^{2}+\beta \frac{f_{\sigma, \eta}^{p}}{W}|\nabla H|^{2},
$$

which gives us the estimate

$$
\int \frac{2 Z}{W} f_{\sigma, \eta}^{p} \leq \frac{C p}{\beta} \int f_{\sigma, \eta}^{p-2}\left|\nabla f_{\sigma, \eta}\right|^{2}+C(1+\beta p) \int \frac{f_{\sigma, \eta}^{p}}{W}|\nabla H|^{2} .
$$

Using the estimate

$$
Z \geq 2 \eta W^{2}-K_{\eta} H^{3}
$$

gives the desired estimate.

Using this Poincaré type inequality, we get the following proposition.

Proposition 6.8. There exists constants $c_{4}, c_{5}$ such that for any $p \geq c_{4}, 0<\sigma<$ $1 / c_{5} \sqrt{p}$ and some constant $K_{2}>0$,

$$
\frac{d}{d t} \int_{\mathcal{M}_{t}}\left(f_{\sigma, \eta}\right)_{+}^{p} d \mu \leq K_{2}\left|\mathcal{M}_{0}\right|
$$

Proof. Consider the evolution equation,

$$
\begin{aligned}
\partial_{t} \int_{\mathcal{M}_{t}} f_{+}^{p} & +p(p-1) \int_{\mathcal{M}_{t}} f_{+}^{p-2}\left|\nabla f_{+}\right|^{2}+\frac{\varepsilon}{12} p \int_{\mathcal{M}_{t}} W^{\sigma-1}|\nabla H|^{2} f_{+}^{p-1} \\
& \leq 4 p a(1-\sigma) \int_{\mathcal{M}_{t}} \frac{H}{W}|\nabla H|\left|\nabla f_{+}\right| f_{+}^{p-1}+\int\left(2 \sigma p\left(|A|^{2}+n K\right)-n K \varepsilon\right) f_{+}^{p} .
\end{aligned}
$$

The term

$$
4 p a(1-\sigma) \int_{\mathcal{M}_{t}} \frac{H}{W}|\nabla H|\left|\nabla f_{+}\right| f_{+}^{p-1}
$$

may be split up as in the previous proposition. Using Young's inequality,

$$
2 \frac{f_{\sigma, \eta}^{p-1}}{\sqrt{W}}|\nabla H|\left|\nabla f_{\sigma, \eta}\right| \leq \frac{f_{\sigma, \eta}^{p-2}}{\beta}\left|\nabla f_{\sigma, \eta}\right|^{2}+\beta \frac{f_{\sigma, \eta}^{p}}{W}|\nabla H|^{2} .
$$

This may be controlled by the Poincaré type inequality,

$$
\frac{2}{\eta} c_{3} \int_{\mathcal{M}_{t}} W f_{+}^{p} \leq \frac{(p+1)}{\beta} \int_{\mathcal{M}_{t}} f_{+}^{p-2}|\nabla f|^{2}+(1+\beta p) \int \frac{f_{+}^{p}}{W}|\nabla H|^{2}+C \int_{\mathcal{M}_{t}} \frac{H^{3}}{W}|\nabla H|^{2}
$$

and we have the following bound for the term:

$$
\frac{\varepsilon}{12} p \int_{\mathcal{M}_{t}} W^{\sigma-1}|\nabla H|^{2} f_{+}^{p-1} \leq \frac{p}{c_{1}} \int_{\mathcal{M}_{t}} \frac{|\nabla H|^{2}}{W} f_{+}^{p} .
$$


Hence we choose our coefficients as follows:

$$
p \geq \max \left\{2, c_{2}\right\}, \quad \sigma \leq \frac{\eta}{8 c_{1} c_{3} \sqrt{p}},
$$

and choose $\beta=\frac{1}{\sqrt{p}}$, and

$$
\frac{p 2 \sigma c_{3}}{\eta \beta} \leq \frac{2 p}{8 c_{1}} \leq \frac{p(p-1)}{4}
$$

and

$$
\frac{p \sigma c_{3}}{\eta}(1+\beta p) \leq \frac{\sqrt{p}}{8 c_{1}}(1+\sqrt{p}) \leq \frac{p}{2 c_{1}}
$$

Hence we have that

$$
p \sigma \int W f_{+}^{p} \leq \frac{p(p-1)}{4} \int f_{+}^{p-2}|\nabla f|^{2}+\frac{p}{2 c_{1}} \int \frac{f_{+}^{p}}{W}|\nabla H|^{2}+\frac{C \sqrt{p}}{8 c_{1}} \int \frac{H^{3}}{W} f_{+}^{p} .
$$

We deal with the last term as follows. Let

$$
q=\frac{2+\bar{\sigma} p}{1+\bar{p}}, \quad q^{\prime}=2 \bar{\sigma} p, \quad B=(p q \sigma)^{\frac{1}{q}} .
$$

We have the following:

$$
\begin{aligned}
\frac{C \sqrt{p}}{8 c_{1}} \frac{H^{3}}{W} & \leq \frac{C^{\prime} \sqrt{p}}{8 c_{1}} \sqrt{W} \\
& \leq \sqrt{W}^{-\bar{\sigma} p} B \sqrt{W}^{1+\bar{\sigma} p} \frac{C^{\prime} \sqrt{p}}{8 B c_{1}} \\
& \leq \sqrt{W}^{\bar{\sigma} p}\left(\frac{B^{q} \sqrt{W}^{q(1+\bar{\sigma} p)}}{q}+\frac{1}{q^{\prime}}\left(\frac{C^{\prime} \sqrt{p}}{8 B c_{1}}\right)^{\frac{1}{q^{\prime}}}\right) \\
& \leq p \sigma W+K \sqrt{W}^{-\bar{\sigma} p} \\
& \leq p \sigma W+K W^{-\sigma p} .
\end{aligned}
$$

This gives us

$$
\frac{C \sqrt{p}}{8 c_{1}} \int \frac{H^{3}}{W} f_{+}^{p} \leq p \sigma \int W f_{+}^{p} d \mu+K \int W^{-\sigma p} f_{+}^{p} d \mu .
$$

We use the fact that $\left(\frac{f_{\sigma, \eta}}{W^{\sigma}}\right)^{p} \leq 1$ to get that

$$
2 p \sigma \int W f_{+}^{p} d \mu \leq \frac{p(p-1)}{2} \int W f_{+}^{p} d \mu+\frac{p}{c_{1}} \int \frac{f_{+}^{p}}{W}|\nabla H|^{2}+2 K\left|\mathcal{M}_{t}\right|
$$

which gives us the estimate

$$
\frac{d}{d t} \int f_{+}^{p} d \mu \leq K\left(n, \eta, \alpha_{n}, \beta_{n}, \sigma, p\right)\left|\mathcal{M}_{0}\right|
$$

Using the above proposition, we get the desired estimate by Stampacchia iteration.

Proof of Theorem 6.3. From the $L^{p}$ estimate of the previous proposition we can derive a uniform bound on the supremum of the function $\left(f_{\sigma, \eta}\right)_{+}$. We leave out the details as they are entirely analogous to similar theorems in [3], 4] and [5]. 


\section{Derivative estimates for the Curvature}

In this section we derive pointwise derivative estimates for the curvature in the mean curvature flow of surfaces satisfying (1.1). The estimates here depend only on the maximum of the curvature at a point and not on the maximum of curvature over the entire submanifold. The proof depends on the following gradient estimate:

$$
|\nabla A|^{2} \geq \frac{3}{n+2}|\nabla H|^{2}
$$

which is obtained from the Codazzi equation. In the following, if $n \geq 4$, then the following constant is positive:

$$
\kappa_{n}=\frac{3}{n-2}-\frac{1}{n-1}>0 .
$$

In fact, this only requires $n \geq 3$.

Theorem 7.1. Let $\mathcal{M}_{t}, t \in[0, T)$ be a closed $n$-dimensional mean convex quadratically bounded solution to the mean curvature flow with $n \geq 4$. Then there exists a constant $\gamma_{2}=\gamma_{2}\left(n, \mathcal{M}_{0}\right)$ and a constant $\gamma_{3}=\gamma_{3}\left(n, \mathcal{M}_{0}\right)$ such that the flow satisfies the uniform estimate

$$
|\nabla A|^{2} \leq \gamma_{2}|A|^{4}+\gamma_{3}
$$

for all $t \in[0, T)$.

Proof. Observe that $\frac{3}{n+2}>\frac{1}{n-1}$ if $n \geq 3$. Let us set

$$
\kappa_{n}=\frac{3}{n+2}-\frac{1}{n-1} \text {. }
$$

By the cylindrical estimate there exists $C_{0}$ such that

$$
\left(\frac{1}{n-1}+\kappa_{n}\right) H^{2}-|A|^{2}+C_{0} \geq 0
$$

Consider also the functions,

$$
g_{1}=\left(\frac{1}{n-1}+\kappa_{n}\right) H^{2}-|A|^{2}+2 C_{0}, \quad g_{2}=\frac{3}{n+2} H^{2}-|A|^{2}+2 C_{0} .
$$

Then we have that $g_{2}>g_{1} \geq C_{0}$ and also we have that $g_{i}-2 C_{0}=2\left(g_{i}-C_{0}\right)-g_{i} \geq$ $-g_{i}$ for $i=1,2$. Using the evolution equations for $|A|^{2}, H^{2}$, (2.6), (2.7) we have that

$$
\begin{aligned}
\frac{\partial}{\partial t} g_{1}-\triangle g_{1} & -2\left(\left(\frac{1}{n-1}+\kappa_{n}\right)|\nabla H|^{2}-|\nabla A|^{2}\right)+2\left(|A|^{2}+n K\right)\left(g_{1}-2 C_{0}\right) \\
& +4 n K\left(|A|^{2}-\frac{H^{2}}{n}\right) \\
& \geq 2\left(1-\frac{n+2}{3}\left(\frac{1}{n-1}+\kappa_{n}\right)\right)|\nabla A|^{2}-2|A|^{2} g_{1} \\
& =2 \kappa_{n} \frac{n+2}{3}|\nabla A|^{2}-2|A|^{2} g_{1} .
\end{aligned}
$$

Similarly we have that

$$
\begin{aligned}
\frac{\partial}{\partial t} g_{2}-\triangle g_{2} & =-2\left(\frac{3}{n+2}|\nabla H|^{2}-|\nabla A|^{2}\right)+2|A|^{2}\left(g_{2}-2 C_{0}\right)+4 n K\left(|A|^{2}-\frac{H^{2}}{n}\right) \\
& \geq-2|A|^{2} g_{2} .
\end{aligned}
$$


Note that as $|A|^{2} \geq \frac{1}{n} H^{2} \geq c>0$ as lower bounds on the mean curvature are preserved by the flow there exists a constant $c$ such that $|A|^{2} \leq c|A|^{4}$ where $c$ depends only on the initial data $\mathcal{M}_{0}$. Furthermore, applying this to the evolution equation (2.9), we have the evolution equation for the derivative of the second fundamental form,

$$
\begin{aligned}
\frac{\partial}{\partial t}|\nabla A|^{2}-\triangle|\nabla A|^{2} & \leq-2\left|\nabla^{2} A\right|^{2}+c_{n}^{\prime}|A|^{2}|\nabla A|^{2}+d_{n}^{\prime}|A||\nabla A|^{2} \\
& -2\left|\nabla^{2} A\right|^{2}+c_{n}|A|^{2}|\nabla A|^{2}
\end{aligned}
$$

for a constant $c_{n}$ depending only on $n$. The remainder of the proof is then entirely analogous to the proof of Theorem 6.1 in $[8]$.

\section{REFERENCES}

[1] Fernando Codá Marques, Deforming three-manifolds with positive scalar curvature, Ann. of Math. (2) 176 (2012), no. 2, 815-863, DOI 10.4007/annals.2012.176.2.3. MR2950765

[2] Richard S. Hamilton, Three-manifolds with positive Ricci curvature, J. Differential Geom. 17 (1982), no. 2, 255-306. MR664497 (84a:53050)

[3] Gerhard Huisken, Flow by mean curvature of convex surfaces into spheres, J. Differential Geom. 20 (1984), no. 1, 237-266. MR772132 (86j:53097)

[4] Gerhard Huisken, Contracting convex hypersurfaces in Riemannian manifolds by their mean curvature, Invent. Math. 84 (1986), no. 3, 463-480, DOI 10.1007/BF01388742. MR837523 (87f:53066)

[5] Gerhard Huisken, Deforming hypersurfaces of the sphere by their mean curvature, Math. Z. 195 (1987), no. 2, 205-219, DOI 10.1007/BF01166458. MR892052 (88d:53058)

[6] Gerhard Huisken, Local and global behaviour of hypersurfaces moving by mean curvature, Differential geometry: partial differential equations on manifolds (Los Angeles, CA, 1990), Proc. Sympos. Pure Math., vol. 54, Amer. Math. Soc., Providence, RI, 1993, pp. 175-191. MR:1216584(94c:58037)

[7] Gerhard Huisken and Carlo Sinestrari, Convexity estimates for mean curvature flow and singularities of mean convex surfaces, Acta Math. 183 (1999), no. 1, 45-70, DOI 10.1007/BF02392946. MR1719551 (2001c:53094)

[8] Gerhard Huisken and Carlo Sinestrari, Mean curvature flow with surgeries of two-convex hypersurfaces, Invent. Math. 175 (2009), no. 1, 137-221, DOI 10.1007/s00222-008-0148-4. MR2461428(2010a:53138)

[9] H. Blaine Lawson Jr., Local rigidity theorems for minimal hypersurfaces, Ann. of Math. (2) 89 (1969), 187-197. MR0238229(38 \#6505)

[10] J. H. Michael and L. M. Simon, Sobolev and mean-value inequalities on generalized submanifolds of $R^{n}$, Comm. Pure Appl. Math. 26 (1973), 361-379. MR0344978 (49 \#9717)

[11] Guido Stampacchia, Èquations elliptiques du second ordre à coefficients discontinus (French), Séminaire de Mathématiques Supérieures, No. 16 (Été, 1965), Les Presses de l'Université de Montréal, Montreal, Que., 1966. MR.0251373 (40 \#4603)

[12] Ben Weinkove, Singularity formation in the Yang-Mills flow, Calc. Var. Partial Differential Equations 19 (2004), no. 2, 211-220, DOI 10.1007/s00526-003-0217-x. MR2034580 (2005b:53111)

Mathematics Institute, Zeeman Bullding, University of Warwick, Coventry, CV4 7AL, United KINGDOM

E-mail address: H.T.Nguyen@warwick.ac.uk

Current address: The School of Mathematics and Physics, The University of Queensland, Brisbane 4072, Australia

E-mail address: huy.nguyen@maths.uq.edu.au 